\title{
SOME WEIGHTED GROUP ALGEBRAS ARE OPERATOR ALGEBRAS
}

\author{
HUN HEE LEE, EBRAHIM SAMEI, AND NICO SPRONK
}

\begin{abstract}
Let $G$ be a finitely generated group with polynomial growth, and let $\omega$ be a weight, i.e. a sub-multiplicative function on $G$ with positive values. We study when the weighted group algebra $\ell^{1}(G, \omega)$ is isomorphic to an operator algebra. We show that $\ell^{1}(G, \omega)$ is isomorphic to an operator algebra if $\omega$ is a polynomial weight with large enough degree or an exponential weight of order $0<\alpha<1$. We will demonstrate the order of growth of $G$ plays an important role in this question. Moreover, the algebraic centre of $\ell^{1}(G, \omega)$ is isomorphic to a $Q$-algebra and hence satisfies a multi-variable von Neumann inequality. We also present a more detailed study of our results when $G$ is the $d$-dimensional integers $\mathbb{Z}^{d}$ and 3-dimensional discrete Heisenberg group $\mathbb{H}_{3}(\mathbb{Z})$. The case of the free group with two generators will be considered as a counter example of groups with exponential growth.
\end{abstract}

\section{INTRODUCTION}

The motivation for this paper was originated from a result of Varopoulos which states that certain weighted group algebras on integers are isomorphic to $Q$-algebras [19]. We recall that a commutative Banach algebra is called a $Q$-algebra if it is a quotient of a uniform algebra. There are interesting (and non-trivial) classes of Banach algebras which are isomorphic to $Q$-algebras. For instance, it is shown in [19] and [6] that the spaces $\ell^{p}(1 \leq p \leq \infty)$ with pointwise product are isomorphic to $Q$-algebras. The case of the Schatten Spaces $S_{p}, 1 \leq p \leq \infty$, endowed with the Schur product, has been considered by many researchers ([11] and [13]), and it has been very recently answered in full generality $([3])$.

Let $G$ be a discrete group, and let $\omega: G \rightarrow(0, \infty)$ be a weight on $G$, i.e.

$$
\omega(x y) \leq \omega(x) \omega(y), \quad x, y \in G .
$$

The weighted group algebra $\ell^{1}(G, \omega)$ is the convolution algebra of functions $f$ on $G$ such that $\|f\|_{\ell^{1}(G, \omega)}=\sum_{x \in G}|f(x)| \omega(x)<\infty$. Varopoulos showed that in the case where $G=\mathbb{Z}$ and $\omega_{\alpha}(n)=(1+|n|)^{\alpha}(\alpha \geq 0), \ell^{1}\left(\mathbb{Z}, \omega_{\alpha}\right)$ is isomorphic to a $Q$-algebra if and only if $\alpha>1 / 2$.

We would like to extend Varopoulos's result to other classes of weighted group algebras, possibly on non-abelian groups. However, group algebras are non-commutative in general, so that we can not hope for them to be isomorphic to $Q$-algebras. Instead, we would like to investigate whether a weighted group algebra is isomorphic to an operator algebra. Recall that an operator algebra is a closed subalgebra of $B(H)$, the algebra of all bounded operators on a Hilbert space $H$. Note that any $Q$-algebra is an operator algebra ([6, Theorem 1.1]). In the proof, Varopoulos actually proved that $\ell^{1}\left(\mathbb{Z}, \omega_{\alpha}\right)$ satisfies one of the sufficient conditions to be isomorphic to a $Q$-algebra, namely it is an injective algebra. Recall that a Banach algebra $\mathcal{A}$

1991 Mathematics Subject Classification. Primary 22D15, 47L30; Secondary 47L25.

Key words and phrases. Weighted group algebras, operator algebras, groups with polynomial growth, Littlewood multipliers.

Hun Hee Lee was supported by Basic Science Research Program through the National Research Foundation of Korea(NRF) funded by the Ministry of Education, Science and Technology (2010- 0015222). Ebrahim Samei was supported by NSERC under grant no. 366066-09. Nico Spronk was supported by NSERC under grant no. 312515-05. 
is called an injective algebra if the algebra multiplication map $m$ extends to a bounded map on the injective tensor product:

$$
m: \mathcal{A} \otimes_{\varepsilon} \mathcal{A} \rightarrow \mathcal{A}
$$

In this paper we also focus on the case where $\ell^{1}(G, \omega)$ becomes an injective algebra. Using a Littlewood multiplier argument we will show that $\ell^{1}(G, \omega)$ is an injective algebra, and consequently is isomorphic to an operator algebra, if $G$ is a finitely generated group with polynomial growth and $\omega$ is a polynomial weight with a large enough degree or a certain exponential weight. Such weights will be defined later in this paper.

This paper is organized as follows. In Section 2.1 we recall several basic facts about injective algebras and $Q$-algebras. In Section 2.2 we give an equivalence condition for $\ell^{1}(G, \omega)$ to be isomorphic to an operator algebra. In Section 2.3 we recall the definitions of Littlewood multipliers and its consequences. In Section 2.4, we give the necessary background on finitely generated groups with polynomial growth and how one can use the length function to define various weights such as polynomial and exponential weights on these groups. In Sections 3.1 and 3.2, we will show our main results, namely the case where $\ell^{1}(G, \omega)$ is isormophic to an operator algebra. Moreover, we will check that the algebraic centre of $\ell^{1}(G, \omega)$ is a $Q$-algebra in this case, and hence, it satisfies the $(\delta, L)$-multi-variable von Neumann inequality (see Section 3.1). We also find estimates for the upper bound of the norm of the multiplication map of the algebra for various weights and use them to determine concrete values of $\delta$ and $L$.

Finally, in Section 5, we apply our techniques to study the cases when $G$ is the $d$-dimensional integers $\mathbb{Z}^{d}$ or 3 -dimensional discrete Heisenberg group $\mathbb{H}_{3}(\mathbb{Z})$. The case of the free group with two generators will be examined to give a reasonable explanation why we mainly focus on groups with polynomial growth.

\section{Preliminaries}

In this paper, all our groups are discrete.

2.1. $p$-summing algebras, injective algebras and $Q$-algebras. We first recall some definitions. Let $X$ and $Y$ be Banach spaces. For $1 \leq p<\infty$, a sequence $\left(x_{n}\right)_{n \geq 1} \subset X$ is called $p$-summable (resp. weakly $p$-summable) if

$$
\left\|\left(x_{n}\right)\right\|_{p}=\left(\sum_{n \geq 1}\left\|x_{n}\right\|^{p}\right)^{\frac{1}{p}}<\infty . \quad\left(\operatorname{resp} .\left\|\left(x_{n}\right)\right\|_{p}^{w}=\sup _{\varphi \in B_{X^{*}}}\left(\sum_{n \geq 1}\left|\varphi\left(x_{n}\right)\right|^{p}\right)^{\frac{1}{p}}<\infty .\right)
$$

The Chevet-Saphar tensor norms on the algebraic tensor product $X \otimes Y$ are defined by

$$
g_{p}(u)=\inf \left\{\left\|\left(x_{j}\right)\right\|_{p}\left\|\left(y_{j}\right)\right\|_{p^{\prime}}^{w}: u=\sum_{j=1}^{n} x_{j} \otimes y_{j}, x_{j} \in X, y_{j} \in Y\right\},
$$

where $p^{\prime}$ is the conjugate index of $p$. We denote the completion of $\left(X \otimes Y, g_{p}\right)$ by $X \otimes_{g_{p}} Y$.

We say that a linear map $T: X \rightarrow Y$ is $p$-summing if there is a constant $C>0$ such that

$$
\left\|\left(T x_{n}\right)\right\|_{p} \leq C\left\|\left(x_{n}\right)\right\|_{p}^{w}
$$

for any sequence $\left(x_{n}\right)_{n \geq 1} \subset X$. We denote the infimum of such $C$ by $\pi_{p}(T)$, and $\Pi_{p}(X, Y)$ refers to the Banach space of all $p$-summing maps with the norm $\pi_{p}(\cdot)$. It is well-known that we have the following isometry

$$
\left(X \otimes_{g_{p}} Y\right)^{*} \cong \Pi_{p^{\prime}}\left(Y, X^{*}\right), A \otimes B \mapsto T
$$


where $A \in X^{*}, B \in Y^{*}$ and

$$
T y=\langle y, B\rangle A, \quad(x \in X, y \in Y) .
$$

See [17, Chapter 6] for the details of $p$-summing maps and Chevet-Saphar tensor norms.

A standard Banach space theory ([17, Proposition 3.22] and [?, Corollary 9.5]) tells us that we have the following isometry

$$
\left(\ell^{1}(G) \otimes_{\varepsilon} \ell^{1}(G)\right)^{*} \cong \Pi_{1}\left(\ell^{1}(G), \ell^{\infty}(G)\right), A \otimes B \mapsto S .
$$

One more standard fact we will use later is that the composition of two 2-summing maps is a 1-summing map (actually, a nuclear map). More precisely, let $T: X \rightarrow Y$ and $S: Y \rightarrow Z$ be 2 -summing maps between Banach spaces, then $S \circ T$ is 1-summing with

$$
\pi_{1}(S \circ T) \leq \pi_{2}(S) \pi_{2}(T) .
$$

We say that a Banach algebra $\mathcal{A}$ is a $p$-summing algebra if the algebra multiplication map $m$ extends to a bounded map

$$
m: \mathcal{A} \otimes_{g_{p}} \mathcal{A} \rightarrow \mathcal{A} .
$$

Theorem 2.1. (Tonge, [7, Theorem 18.19]) Every 2-summing algebra is isomorphic to an operator algebra.

Corollary 2.2. Every injective algebra is isomorphic to an operator algebra.

Proof. Recall that the injective tensor product is the minimal among Banach space tensor products, so that the formal identity $\mathcal{A} \otimes_{g_{2}} \mathcal{A} \rightarrow \mathcal{A} \otimes_{\varepsilon} \mathcal{A}$ is a contraction for a Banach algebra $\mathcal{A}$. Thus we can conclude that every injective algebra is a 2 -summing algebra, which gives the conclusion we wanted.

Definition 2.3. Let $m$ be the algebra multiplication of a Banach algebra $\mathcal{A}$. In the case that $\mathcal{A}$ is an injective algebra, we will denote

$$
\|m\|_{\varepsilon}:=\left\|m: \mathcal{A} \otimes_{\varepsilon} \mathcal{A} \rightarrow \mathcal{A}\right\| .
$$

We say a Banach algebra $\mathcal{A}$ is a $Q$-algebra if it is a quotient of a uniform algebra, which is automatically a commutative algebra. $Q$-algebras are characterized by a von Neumann type inequality [4, Section 5.4.3(2)].

Theorem 2.4. Let $\mathcal{A}$ be a commutative Banach algebra. Then $\mathcal{A}$ is isometrically isomorphic to a $Q$-algebra if and only if we have

$$
\left\|p\left(a_{1}, \ldots, a_{n}\right)\right\| \leq\|p\|_{\infty}
$$

for any $n \in N,\left\{a_{1}, \ldots, a_{n}\right\} \subset A$ with norm $\leq 1$ and every polynomial $p$ in $n$ variables without constant terms, where

$$
\|p\|_{\infty}=\sup \left\{\left|p\left(z_{1}, \ldots, z_{n}\right)\right|\left|z_{i}\right| \leq 1, i=1, \ldots, n\right\} .
$$

Motivated by the above we give the following definition.

Definition 2.5. Let $A$ be a commutative Banach algebra. Then $A$ is said to satisfy multivariable $(\delta, L)$-von Neumann inequality provided that for every $n \in N$, every set of $n$ elements $\left\{a_{1}, \ldots, a_{n}\right\} \subset A$ with $\left\|a_{i}\right\| \leq \delta(i=1, \ldots, n)$, and every polynomial $p$ in $n$ variables without constant terms, we have

$$
\left\|p\left(a_{1}, \ldots, a_{n}\right)\right\| \leq L\|p\|_{\infty} .
$$


Every commutative injective algebras are $Q$-algebras ([19]). Actually, a commutative Banach algebra is an injective algebra if and only if it is isomorphic to a quotient of a uniform algebra by a complemented ideal ([20]). A more qualitative result can be found in [4] using a modern language of operator spaces.

Theorem 2.6. (Blecher/Le Merdy, [4, Theorem 5.4.5, Corollary 5.4.11]) Let $\mathcal{A}$ be a commutative injective algebra with the multiplication map $m$. Then $\mathcal{A}$ satisfies the multi-variable $(\delta, L)$-von Neuman inequality with

$$
\delta=\frac{1}{\left(1+\|m\|_{\varepsilon}\right) e} \text { and } L=1 .
$$

2.2. Weighted group algebras. Let $G$ be a group, and let $\omega: G \rightarrow(0, \infty)$ be a weight on $G$, i.e.

$$
\omega(x y) \leq \omega(x) \omega(y), \quad(x, y \in G) .
$$

The weighted group algebra $\ell^{1}(G, \omega)$ is the convolution algebra of functions $f$ on $G$ such that $\|f\|_{\ell^{1}(G, \omega)}=\sum_{x \in G}|f(x)| \omega(x)<\infty$. Using the natural duality $\ell^{1}(G)^{*}=\ell^{\infty}(G)$, we can show that $\ell^{1}(G, \omega)^{*}=\ell^{\infty}\left(G, \omega^{-1}\right)$, where

$$
\ell^{\infty}\left(G, \omega^{-1}\right)=\left\{\varphi \mid \varphi \omega^{-1} \in \ell^{\infty}(G)\right\}
$$

with

$$
\|\varphi\|_{\ell^{\infty}\left(G, \omega^{-1}\right)}=\left\|\varphi \omega^{-1}\right\|_{\infty} .
$$

In Section 2.1, we showed that every injective Banach algebra is isomorphic to an operator algebra. As we will show in Theorem 2.8 below, the converse of the preceding statement is also true in the case of weighted group algebras. However, this requires some operator space knowledge including the Haagerup tensor product $\otimes_{h}$ of operator spaces. We refer the reader to [4] or [15] for references. We first recall the following form of the celebrated Grothendieck's theorem.

Theorem 2.7. If we equip $\ell^{1}(G)$ with its $M A X$ operator space structure, then the formal identity $i d: \ell^{1}(G) \otimes_{\varepsilon} \ell^{1}(G) \rightarrow \ell^{1}(G) \otimes_{h} \ell^{1}(G)$ has norm $\leq K_{G}$, where $K_{G}$ is the Grothendieck's constant.

Proof. See [4, (1.47), (A.7)] and [16, (3.11)].

Theorem 2.8. Let $G$ be a group, and let $\omega$ be a weight on $G$. Then $\ell^{1}(G, \omega)$ is an injective Banach algebra if and only if it is isomorphic to an operator algebra.

Proof. The necessary part has been proven in Corollary 2.2. For the sufficient part, suppose that there is an operator algebra $B \subseteq B(H)$ and a bounded algebra isomorphism $\psi: \ell^{1}(G, \omega) \rightarrow B$. This, in particular, implies that $\psi: \ell^{1}(G, \omega) \rightarrow B$ is completely bounded when $\ell^{1}(G, \omega)$ is given its MAX operator space structure. Now since from [4, Theorem 2.3.2], the multiplication map $m: B \otimes_{h} B \rightarrow B$ is completely contractive, we have the following bounded map:

$$
\psi^{-1} \circ m \circ(\psi \otimes \psi): \ell^{1}(G, \omega) \otimes_{h} \ell^{1}(G, \omega) \rightarrow \ell^{1}(G, \omega)
$$

But it is easy to see that $\psi^{-1} \circ m \circ(\psi \otimes \psi)$ is exactly the multiplication map

$$
m: \ell^{1}(G, \omega) \otimes_{h} \ell^{1}(G, \omega) \rightarrow \ell^{1}(G, \omega) .
$$

On the other hand, since the mapping

$$
\ell^{1}(G, \omega) \rightarrow \ell^{1}(G), f \mapsto f \omega,
$$

is a complete isometric surjection (here we have again given MAX operator space structure to both $\ell^{1}(G, \omega)$ and $\left.\ell^{1}(G)\right)$, it follows from the Grothendieck's theorem (Theorem 2.7) that the 
formal identity $i d: \ell^{1}(G, \omega) \otimes_{\varepsilon} \ell^{1}(G, \omega) \rightarrow \ell^{1}(G, \omega) \otimes_{h} \ell^{1}(G, \omega)$ has norm $\leq K_{G}$. This implies that the multiplication map $\ell^{1}(G, \omega) \otimes_{\varepsilon} \ell^{1}(G, \omega) \rightarrow \ell^{1}(G, \omega)$ is bounded, and so, $\ell^{1}(G, \omega)$ is injective.

2.3. Littlewood multiplier. Let $G$ be a (discrete) group. We let the space of Littlewood multipliers, denoted by $T^{2}(G)$, to be all the functions $f: G \times G \rightarrow \mathbb{C}$ for which there are functions $f_{1}, f_{2}: G \times G \rightarrow \mathbb{C}$ such that

$$
f(s, t)=f_{1}(s, t)+f_{2}(s, t) \quad(s, t \in G),
$$

and

$$
\sup _{t \in G} \sum_{s \in G}\left|f_{1}(s, t)\right|^{2}<\infty, \sup _{s \in G} \sum_{t \in G}\left|f_{2}(s, t)\right|^{2}<\infty .
$$

We equip this space with the norm

$$
\|f\|_{T_{2}(G)}=\inf \left\{\sup _{t \in G}\left(\sum_{s \in G}\left|f_{1}(s, t)\right|^{2}\right)^{1 / 2}+\sup _{s \in G}\left(\sum_{t \in G}\left|f_{2}(s, t)\right|^{2}\right)^{1 / 2}\right\},
$$

where infimum is taken over all possible decompositions. Note that the term "Littlewood functions" have been used for $T^{2}(G)$ in the literature, but we would like to use the term "Littlewood multipliers" instead since it explains the meaning of $T^{2}(G)$ better.

It follows easily that $T^{2}(G)$, with the action of pointwise multiplication, is a symmetric Banach $\ell^{\infty}(G \times G)$-module. Indeed, we have the following contraction.

$$
\ell^{\infty}(G \times G) \otimes_{\gamma} T^{2}(G) \rightarrow T^{2}(G), f \otimes g \mapsto f g,
$$

where $\otimes_{\gamma}$ is the projective tensor product of Banach spaces. Moreover, we have the following bounded embedding which is well-known to experts but we have presented its proof for the sake of completeness.

Proposition 2.9. Let $G$ be a discrete group, and let $I: T_{2}(G) \rightarrow\left(\ell^{1}(G) \otimes_{\varepsilon} \ell^{1}(G)\right)^{*}$ be the formal identity. Then we have

$$
\|I\| \leq K_{G}
$$

Proof. For simplicity, we write $\ell^{1}$ instead of $\ell^{1}(G), \ell^{2}$ instead of $\ell^{2}(G)$ and $\ell^{\infty}$ instead of $\ell^{\infty}(G)$. We first note that since $\ell^{2}$ is reflexive, we have the following isometric isomorphisms

$$
B\left(\ell^{1}, \ell^{2}\right) \cong\left(\ell^{1} \otimes^{\gamma} \ell^{2}\right)^{*} \cong\left(\ell^{1}\left(\ell^{2}\right)\right)^{*} \cong \ell^{\infty}\left(\ell^{2}\right),
$$

where $\ell^{1}\left(\ell^{2}\right)$ and $\ell^{\infty}\left(\ell^{2}\right)$ are Banach spaces of $\ell^{2}$-valued 1-summable functions and bounded functions, respectively. Now let $f_{1}: G \times G \rightarrow \mathbb{C}$ be a function with

$$
\alpha:=\sup _{t \in G}\left(\sum_{s \in G}\left|f_{1}(s, t)\right|^{2}\right)^{1 / 2}<\infty .
$$

Then, by (2.4), the associated linear map $u: \ell^{1} \rightarrow \ell^{2}, g \mapsto u(g)$ given by

$$
u(g)(t)=\sum_{s \in G} g(s) f_{1}(s, t)
$$

has the norm $\|u\|=\alpha$ and $I\left(f_{1}\right)$ corresponds to $i d_{2, \infty} \circ u$, where $i d_{2, \infty}: \ell^{2} \rightarrow \ell^{\infty}$ is the formal identity. Now we recall that

$$
\left(\ell^{1}(G) \otimes_{\varepsilon} \ell^{1}(G)\right)^{*} \cong \Pi_{1}\left(\ell^{1}(G), \ell^{\infty}(G)\right)
$$

and

$$
\left(\ell^{1}(G) \otimes_{h} \ell^{1}(G)\right)^{*} \cong \Gamma_{2}\left(\ell^{1}(G), \ell^{\infty}(G)\right)
$$


the space of 2-factorable operators, as Banach spaces ([15, Proposition 5.16], [7, Chapter 7]). Then, by Grothendieck's theorem (Theorem 2.7), we have

$$
\left\|I\left(f_{1}\right)\right\|=\pi_{1}\left(i d_{2, \infty} \circ u\right) \leq K_{G} \cdot \gamma_{2}\left(i d_{2, \infty} \circ u\right) \leq K_{G} \cdot \alpha,
$$

where $\gamma_{2}(\cdot)$ is the 2 -factorable norm. Similarly, for $f_{2}: G \times G \rightarrow \mathbb{C}$ with

$$
\beta:=\sup _{s \in G}\left(\sum_{t \in G}\left|f_{1}(s, t)\right|^{2}\right)^{1 / 2}<\infty
$$

we get $\left\|I\left(f_{2}\right)\right\| \leq K_{G} \cdot \beta$, which gives the desired result.

2.4. Groups with polynomial growth. Let $G$ be a finitely generated group with a fixed finite symmetric generating set $F$ with the identity of the group $G$. $G$ is said to have polynomial growth if there exists a polynomial $f$ such that

$$
\left|F^{n}\right| \leq f(n) \quad(n \in \mathbb{N}) .
$$

Here $|S|$ is the cardinality of any $S \subseteq G$ and

$$
F^{n}=\left\{u_{1} \cdots u_{n}: u_{i} \in F, i=1, \ldots, n\right\},
$$

where $\left\{u_{i}\right\}_{i=1}^{n}$ is the set of generators. The least degree of any polynomial satisfying the above relation is called the order of growth of $G$ and it is denoted by $d(G)$. It can be shown that the order of growth of $G$ does not depend on the symmetric generating set $F$, i.e. it is a universal constant for $G$.

It is immediate that finite groups are of polynomial growth. More generally, every $G$ with the property that the conjugacy class of every element in $G$ is finite has polynomial growth [12, Theorem 12.5.17]. Also every nilpotent group (hence an abelian group) has polynomial growth [12, Theorem 12.5.17]. A deep result of M. Gromov [9] states that every finitely generated group with polynomial growth is virtually nilpotent i.e. it has a nilpotent subgroup of finite index. Moreover, there is a polynomial $f$ and a constant $0<\lambda \leq 1$ such that

$$
\lambda f(n) \leq\left|F^{n}\right| \leq f(n) \text { for all } n \in \mathbb{N},
$$

where $\operatorname{deg} f=d(G)$. If we further assume that $G$ is nilpotent, then by the Bass-Guivarch formula ([1], 10]), we can actually compute the order of growth of $G$. More precisely, let $G$ be a finitely generated nilpotent group with lower central series

$$
G=G_{1} \supseteq G_{2} \supseteq \ldots \supseteq G_{m}=\{e\} .
$$

In particular, the quotient group $G_{k} / G_{k+1}$ is a finitely generated abelian group. Then the order of growth of $\mathrm{G}$ is

$$
d(G)=\sum_{k=1}^{m-1} k \operatorname{rank}\left(G_{k} / G_{k+1}\right),
$$

where rank denotes the rank of an abelian group, i.e. the largest number of independent and torsion-free elements of the abelian group.

Using the generating set $F$ of $G$ we can define a length function $\tau_{F}: G \rightarrow[0, \infty)$ by

$$
\tau_{F}(x)=\inf \left\{n \in \mathbb{N}: x \in F^{n}\right\} \text { for } x \neq e, \quad \tau_{F}(e)=0 .
$$

When there is no fear of ambiguity, we write $\tau$ instead of $\tau_{F}$. It is straightforward to verify that $\tau$ is a subadditive function on $G$, i.e.

$$
\tau(x y) \leq \tau(x)+\tau(y) \quad(x, y \in G) .
$$


Note that since $F$ is symmetric, for every $x \in G, \tau(x)=\tau\left(x^{-1}\right)$. If we combine this fact with (2.8), then a straightforward calculation shows that

$$
|\tau(x)-\tau(y)| \leq \tau(x y) \leq \tau(x)+\tau(y) \quad(x, y \in G) .
$$

We can use $\tau$ to define various weights on $G$. More precisely, for every $0 \leq \alpha \leq 1, \beta \geq 0$, and $C>0$, we can define the polynomial weight $\omega_{\beta}$ on $G$ of order $\beta$ by

$$
\omega_{\beta}(x)=(1+\tau(x))^{\beta} \quad(x \in G),
$$

and the exponential weight $\sigma_{\alpha, C}$ on $G$ of order $(\alpha, C)$ by

$$
\sigma_{\alpha, C}(x)=e^{C \tau(x)^{\alpha}} \quad(x \in G) .
$$

\section{WEIGHTED GROUP ALGEBRAS ISOMORPHIC TO OPERATOR ALGEBRAS}

In this section, we will use $G$ to denote a finitely generated infinite group with polynomial growth. $F$ is a fixed symmetric generating set of $G$ with the identity and $f$ and $\lambda$ refer to the polynomial and the constant satisfying (2.5).

3.1. The case of polynomial weights. For some weight $\omega: G \rightarrow(\delta, \infty)$ with $\delta>0$, we would like to check whether $\ell^{1}(G, \omega)$ is an injective algebra. In order to do that we recall the co-multiplication

$$
\Gamma: \ell^{\infty}(G) \rightarrow \ell^{\infty}(G \times G), f \mapsto \Gamma f
$$

with $\Gamma f(s, t)=f(s t), s, t \in G$. Let $\Gamma_{\omega}: \ell^{\infty}\left(G, \omega^{-1}\right) \rightarrow \ell^{\infty}\left(G \times G, \omega^{-1} \times \omega^{-1}\right)$ be the extension of $\Gamma$ to $\ell^{\infty}\left(G, \omega^{-1}\right)$. Consider the isometries

$$
P: \ell^{\infty}(G) \rightarrow \ell^{\infty}\left(G, \omega^{-1}\right), f \mapsto f \omega
$$

and

$$
R: \ell^{\infty}\left(G \times G, \omega^{-1} \times \omega^{-1}\right) \rightarrow \ell^{\infty}(G \times G), F \mapsto F \cdot\left(\omega^{-1} \times \omega^{-1}\right) .
$$

We define the operator $\tilde{\Gamma}: \ell^{\infty}(G) \rightarrow \ell^{\infty}(G \times G)$ so that the following diagram commutes:

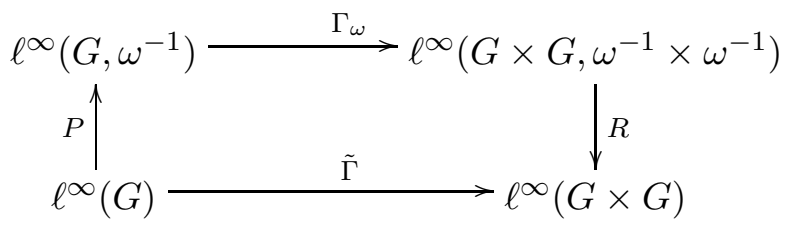

Hence

$$
\tilde{\Gamma}(f)=\Omega \Gamma(f) \quad\left(f \in \ell^{\infty}(G)\right)
$$

where

$$
\Omega:=\frac{\Gamma(\omega)}{\omega \times \omega} .
$$

Now $\ell^{1}(G, \omega)$ is an injective algebra if and only if the multiplication map

$$
m: \ell^{1}(G, \omega) \otimes_{\varepsilon} \ell^{1}(G, \omega) \rightarrow \ell^{1}(G, \omega)
$$

is bounded, or equivalently, $\tilde{\Gamma}$ extends to a bounded map

$$
\tilde{\Gamma}: \ell^{\infty}(G) \rightarrow\left(\ell^{1}(G) \otimes_{\varepsilon} \ell^{1}(G)\right)^{*} .
$$

Note that we have

$$
\|m\|_{\varepsilon}=\|\tilde{\Gamma}\|
$$


An application of Littlewood multiplier argument gives the following positive results on the weighted group algebra $\ell^{1}\left(G, \omega_{\beta}\right)$, where $\omega_{\beta}$ is the polynomial weight defined in (2.10).

Theorem 3.1. $\ell^{1}\left(G, \omega_{\beta}\right)$ is an injective algebra if one of the following conditions holds:

(i) $\lambda=1$ and $\beta>\frac{d(G)}{2}$;

(ii) $0<\lambda<1$ and $\beta>\frac{d(G)+1}{2}$.

Moreover, we have

$$
\|m\|_{\varepsilon} \leq K_{G} \min \left\{1,2^{\beta-1}\right\}\left[1+\sum_{n=1}^{\infty} \frac{f(n)-\lambda f(n-1)}{(1+n)^{2 \beta}}\right]^{1 / 2} .
$$

Proof. Let $\Omega_{\beta}:=\frac{\Gamma\left(\omega_{\beta}\right)}{\omega_{\beta} \times \omega_{\beta}}$. We will first show that $\Omega_{\beta} \in T^{2}(G)$. For every $x, y \in G$, we have

$$
\begin{aligned}
\Omega_{\beta}(x, y) & =\frac{\omega_{\beta}(x y)}{\omega_{\beta}(x) \omega_{\beta}(y)} \\
& =\frac{(1+\tau(x y))^{\beta}}{(1+\tau(x))^{\beta}(1+\tau(y))^{\beta}} \\
& \leq \frac{(1+\tau(x)+\tau(y))^{\beta}}{(1+\tau(x))^{\beta}(1+\tau(y))^{\beta}} \\
& \leq \frac{A_{\beta}\left[(1+\tau(x))^{\beta}+(1+\tau(y))^{\beta}\right]}{(1+\tau(x))^{\beta}(1+\tau(y))^{\beta}} \\
& =\frac{A_{\beta}}{(1+\tau(x))^{\beta}}+\frac{A_{\beta}}{(1+\tau(y))^{\beta}},
\end{aligned}
$$

where $A_{\beta}=\min \left\{1,2^{\beta-1}\right\}$ and the inequality $(*)$ follows from the classical inequality

$$
(a+b)^{\beta} \leq A_{\beta}\left(a^{\beta}+b^{\beta}\right) \quad(a, b \geq 0) .
$$

Hence there is the function $u \in \ell^{\infty}(G \times G)$ with $\|u\|_{\infty} \leq 1$ such that

$$
\Omega_{\beta}(x, y)=u(x, y)\left[\frac{A_{\beta}}{(1+\tau(x))^{\beta}}+\frac{A_{\beta}}{(1+\tau(y))^{\beta}}\right] \quad(x, y \in G) .
$$

Thus, by the definition of $T^{2}(G)$ and (2.3),

$$
\left\|\Omega_{\beta}\right\|_{T^{2}(G)} \leq A_{\beta}\left(\sum_{x \in G} \frac{1}{(1+\tau(x))^{2 \beta}}\right)^{1 / 2} .
$$

Hence it suffices to see when $\sum_{x \in G} \frac{1}{(1+\tau(x))^{2 \beta}}$ is finite. To see this, from our hypothesis and (2.5), we have

$$
\begin{aligned}
\sum_{x \in G} \frac{1}{(1+\tau(x))^{2 \beta}} & =\sum_{n=0}^{\infty} \sum_{\tau(x)=n} \frac{1}{(1+n)^{2 \beta}} \\
& =1+\sum_{n=1}^{\infty} \sum_{x \in F^{n} \backslash F^{n-1}} \frac{1}{(1+n)^{2 \beta}} \\
& \leq 1+\sum_{n=1}^{\infty} \frac{f(n)-\lambda f(n-1)}{(1+n)^{2 \beta}}
\end{aligned}
$$


where the series in the last line converges if $\lambda=1$ and $2 \beta>d$ or $0<\lambda<1$ and $2 \beta>d+1$. Moreover, in either case, we have

$$
\sum_{x \in G} \frac{1}{(1+\tau(x))^{2 \beta}} \leq 1+\sum_{n=1}^{\infty} \frac{f(n)-\lambda f(n-1)}{(1+n)^{2 \beta}} .
$$

Hence, by Proposition 2.9 and (2.3),

$$
\begin{aligned}
\|\tilde{\Gamma}(f)\|_{\left(\ell^{1}(G) \otimes_{\varepsilon} \ell^{1}(G)\right)^{*}} & \leq K_{G}\|\tilde{\Gamma}(f)\|_{T^{2}(G)} \\
& \leq K_{G}\left\|\Omega_{\beta}\right\|_{T^{2}(G)}\|\Gamma(f)\|_{\infty} \\
& \leq K_{G} A_{\beta}\left[1+\sum_{n=1}^{\infty} \frac{f(n)-\lambda f(n-1)}{(1+n)^{2 \beta}}\right]^{1 / 2}\|f\|_{\infty}
\end{aligned}
$$

for any $f \in \ell^{\infty}(G)$.

3.2. The case of exponential weights. In this section we will study when the weighted group algebra $\ell^{1}\left(G, \sigma_{\alpha, C}\right)$ is an injective algebra, where $\sigma_{\alpha, C}$ is the exponential weight defined in (2.11). If we consider the same additional function $\Omega=\frac{\Gamma(\omega)}{\omega \times \omega}$, then it is not clear this time whether we can split the function into two parts with a suitable square summability. However, we can majorize the function with a similar one coming from a polynomial weight. Let us begin with a technical lemma.

Lemma 3.2. Let $0<\alpha<1, C>0$ and take $\beta \geq \max \left\{1, \frac{6}{C \alpha(1-\alpha)}\right\}$. Define the functions $p:[0, \infty) \rightarrow \mathbb{R}$ and $q:(0, \infty) \rightarrow \mathbb{R}$ by

$$
p(x)=C x^{\alpha}-\beta \ln (1+x) \quad, \quad q(x)=\frac{p(x)}{x} .
$$

Then $p$ is increasing and $q$ is decreasing on $\left[\left(\frac{\beta^{2}}{C \alpha(1-\alpha)}\right)^{1 / \alpha}, \infty\right)$.

Proof. We have

$$
p^{\prime}(x)=C \alpha x^{\alpha-1}-\frac{\beta}{1+x}=\frac{C \alpha x^{\alpha-1}+C \alpha x^{\alpha}-\beta}{1+x} .
$$

Hence $p^{\prime}(x) \geq 0$ if $C \alpha x^{\alpha}-\beta \geq 0$. This implies that

$$
p \text { is increasing on }\left[\left(\frac{\beta}{C \alpha}\right)^{1 / \alpha}, \infty\right) \text {. }
$$

Now consider $q(x)=C x^{\alpha-1}-\frac{\beta \ln (1+x)}{x}$. Then

$$
q^{\prime}(x)=\frac{C(\alpha-1) x^{\alpha}-\frac{\beta x}{1+x}+\beta \ln (1+x)}{x^{2}}=\frac{h(x)-\frac{\beta x}{1+x}}{x^{2}},
$$

where

$$
h(x):=C(\alpha-1) x^{\alpha}+\beta \ln (1+x) .
$$


Hence, in order to find an interval for which $q^{\prime}(x) \leq 0$, it suffices to see when $h(x) \leq 0$. We have

$$
h^{\prime}(x)=\frac{C \alpha(\alpha-1) x^{\alpha}+C \alpha(\alpha-1) x^{\alpha-1}+\beta}{1+x} .
$$

Thus if we put

$$
C_{1}=C \alpha(1-\alpha)
$$

then $h^{\prime}(x) \leq 0$ whenever $-C_{1} x^{\alpha}+\beta \leq 0$, or equivalently, $x \geq\left(\frac{\beta}{C_{1}}\right)^{1 / \alpha}$. Hence

$$
h \text { is decreasing on }\left[\left(\frac{\beta}{C_{1}}\right)^{1 / \alpha}, \infty\right) \text {. }
$$

Now since, by hypothesis, $\beta \geq 1$, we have

$$
\left(\frac{\beta^{2}}{C_{1}}\right)^{1 / \alpha} \geq\left(\frac{\beta}{C_{1}}\right)^{1 / \alpha}
$$

and so by (3.8),

$$
h(x) \leq h\left(\left(\frac{\beta^{2}}{C_{1}}\right)^{1 / \alpha}\right) \quad \text { whenever } \quad x \geq\left(\frac{\beta^{2}}{C_{1}}\right)^{1 / \alpha} .
$$

This implies that if $x \geq\left(\frac{\beta^{2}}{C_{1}}\right)^{1 / \alpha}$, then

$$
\begin{aligned}
h(x) & \leq C(\alpha-1) \frac{\beta^{2}}{C_{1}}+\beta \ln \left(1+\left(\frac{\beta^{2}}{C_{1}}\right)^{1 / \alpha}\right) \\
& =\beta\left[\ln \left(1+\left(\frac{\beta^{2}}{C_{1}}\right)^{1 / \alpha}\right)-\frac{\beta}{\alpha}\right] .
\end{aligned}
$$

On the other hand, since $\beta \geq \frac{6}{C \alpha(1-\alpha)}=\frac{6}{C_{1}}$, we have $\frac{\beta^{2}}{C_{1}} \leq \frac{\beta^{3}}{6}$. Hence, considering the fact that $1 / \alpha>1$,

$$
\begin{aligned}
1+\left(\frac{\beta^{2}}{C_{1}}\right)^{1 / \alpha} & \leq 1+\left(\frac{\beta^{3}}{3 !}\right)^{1 / \alpha} \\
& \leq\left(1+\frac{\beta^{3}}{3 !}\right)^{1 / \alpha} \\
& \leq\left(\sum_{n=0}^{\infty} \frac{\beta^{n}}{n !}\right)^{1 / \alpha} \\
& =e^{\frac{\beta}{\alpha}} .
\end{aligned}
$$

Therefore

$$
\ln \left(1+\left(\frac{\beta^{2}}{C_{1}}\right)^{1 / \alpha}\right)-\frac{\beta}{\alpha} \leq 0
$$

Hence $h(x) \leq 0$ if $x \geq\left(\frac{\beta^{2}}{C_{1}}\right)^{1 / \alpha}$. By (3.7)

$$
q(x) \text { is decreasing on }\left[\left(\frac{\beta^{2}}{C_{1}}\right)^{1 / \alpha}, \infty\right) .
$$

The final result follows from $($ (3.6) $)$ and the fact that $\left(\frac{\beta^{2}}{C_{1}}\right)^{1 / \alpha} \geq\left(\frac{\beta}{C \alpha}\right)^{1 / \alpha}$. 
Theorem 3.3. Suppose that $0<\alpha<1, C>0$, and $\beta \geq \max \left\{1, \frac{6}{C \alpha(1-\alpha)}\right\}$. Let $p$ and $q$ be the functions defined in (3.5) and consider the function $\omega: G \rightarrow(0, \infty)$ defined by

$$
\omega(x)=e^{p(\tau(x))}=e^{\tau(x) q(\tau(x))} \quad(x \in G) .
$$

Then

$$
\omega(x y) \leq M \omega(x) \omega(y) \quad(x, y \in G)
$$

where

$$
M=\max \left\{e^{p(t)-p(s)-p(r)}: t, s, r \in[0,4 K] \cap \mathbb{Z}\right\}
$$

and

$$
K=\left(\frac{\beta^{2}}{C \alpha(1-\alpha)}\right)^{1 / \alpha} .
$$

Proof. By Lemma 3.2, $p$ is increasing and $q$ is decreasing on $[K, \infty)$. We will prove the statement of the theorem considering various cases:

Case I: $\max \{\tau(x), \tau(y)\} \leq 2 K$. In this case, $\tau(x y) \leq \tau(x)+\tau(y) \leq 4 K$. Hence

$$
\frac{\omega(x y)}{\omega(x) \omega(y)}=e^{p(\tau(x y))-p(\tau(x))-p(\tau(y))} \leq M .
$$

Case II: $\max \{\tau(x), \tau(y)\}>2 K$ and $\min \{\tau(x), \tau(y)\} \leq K$. Without loss of generality, we can assume that $\tau(x)>2 K$ and $\tau(y) \leq K$. Then, by (2.9),

$$
\tau(x)+\tau(y) \geq \tau(x y) \geq \tau(x)-\tau(y) \geq 2 K-K=K .
$$

Thus, by Lemma 3.2 ,

$$
\begin{aligned}
\omega(x y) & =e^{p(\tau(x y))} \\
& \leq e^{p(\tau(x)+\tau(y))} \\
& =e^{(\tau(x)+\tau(y)) q(\tau(x)+\tau(y))} \\
& =e^{\tau(x) q(\tau(x)+\tau(y))} e^{\tau(y) q(\tau(x)+\tau(y))} \\
& \leq e^{\tau(x) q(\tau(x))} e^{K q(K)} \\
& =\omega(x) \omega(y) e^{p(K)-p(\tau(y))} \\
& \leq M \omega(x) \omega(y) .
\end{aligned}
$$

Case III: $\min \{\tau(x), \tau(y)\}>K$ and $\tau(x y) \leq K$. In this case, we have

$$
\begin{aligned}
\omega(x) \omega(y) & =e^{p(\tau(x))+p(\tau(y))} \\
& \geq e^{2 p(K)} \\
& =e^{2 p(K)-p(\tau(x y))} \omega(x y) \\
& \geq \frac{1}{M} \omega(x y) .
\end{aligned}
$$

Hence

$$
\omega(x y) \leq M \omega(x) \omega(y)
$$


Case IV: $\min \{\tau(x), \tau(y), \tau(x y)\}>K$. In this case, by Lemma 3.2, we have

$$
\begin{aligned}
\omega(x y) & =e^{p(\tau(x y))} \\
& \leq e^{p(\tau(x)+\tau(y))} \\
& =e^{(\tau(x)+\tau(y)) q(\tau(x)+\tau(y))} \\
& =e^{\tau(x) q(\tau(x)+\tau(y))} e^{\tau(y) q(\tau(x)+\tau(y))} \\
& \leq e^{\tau(x) q(\tau(x))} e^{\tau(y) q(\tau(y)} \\
& \leq \omega(x) \omega(y) .
\end{aligned}
$$

Therefore by comparing the above four cases and considering the fact that $M \geq e^{-p(0)}=1$, it follows that for every $x, y \in G$,

$$
\omega(x y) \leq M \omega(x) \omega(y)
$$

We are now ready to show when the weighted group algebras of exponential weights are injective algebras.

Theorem 3.4. Suppose that $0<\alpha<1$ and $C>0$. Then $\ell^{1}\left(G, \sigma_{\alpha, C}\right)$ is a 2-summing algebra. Moreover, we have

$$
\|m\|_{\varepsilon} \leq K_{G} M 2^{\beta-1}\left[1+\sum_{n=1}^{\infty} \frac{f(n)-\lambda f(n-1)}{(1+n)^{2 \beta}}\right]^{1 / 2}
$$

where

$$
\beta=\max \left\{1, \frac{6}{C \alpha(1-\alpha)}, \frac{d+\left(1-\delta_{1}(\lambda)\right)}{2}\right\}
$$

( $\delta_{1}$ is the Dirac function at 1 ) and $M$ is the constant (depending on $\alpha, \beta$ and $C$ ) defined in (3.10).

Proof. We define a function $\omega: G \rightarrow(0, \infty)$ by

$$
\omega(x)=\frac{\sigma_{\alpha, C}(x)}{\omega_{\beta}(x)}=e^{C \tau(x)^{\alpha}-\beta \ln (1+\tau(x))} \quad(x \in G),
$$

where $\omega_{\beta}$ is the polynomial weight defined in (2.10). Then by Theorem 3.3 ,

$$
\omega(x y) \leq M \omega(x) \omega(y) \quad(x, y \in G),
$$

where $M$ is the constant defined in (3.10). Therefore if we let

$$
\Sigma_{\alpha, C}:=\frac{\Gamma\left(\sigma_{\alpha, C}\right)}{\sigma_{\alpha, C} \times \sigma_{\alpha, C}} \text { and } \Omega_{\beta}:=\frac{\Gamma\left(\omega_{\beta}\right)}{\omega_{\beta} \times \omega_{\beta}},
$$

then

$$
\Sigma_{\alpha, C} \leq M \Omega_{\beta} \leq M\left[\frac{2^{\beta-1}}{(1+\tau(x))^{\beta}}+\frac{2^{\beta-1}}{(1+\tau(y))^{\beta}}\right]
$$

A similar argument to the one presented in the proof of Theorem 3.1 shows that

$$
\|m\|_{\varepsilon} \leq K_{G}\left\|\Sigma_{\alpha, C}\right\|_{T^{2}(G)} \leq K_{G} M 2^{\beta-1}\left[1+\sum_{n=1}^{\infty} \frac{f(n)-\lambda f(n-1)}{(1+n)^{2 \beta}}\right]^{1 / 2} .
$$

In particular, $\ell^{1}\left(G, \sigma_{\alpha, C}\right)$ is an injective algebra.

We can actually show exactly when the weighted group algebras of exponential weight are isomorphic to an operator algebra. 
Theorem 3.5. Suppose that $0 \leq \alpha \leq 1$ and $C>0$. Then $\ell^{1}\left(G, \sigma_{\alpha, C}\right)$ is isomorphic to an operator algebra if and only if $0<\alpha<1$.

Proof. The case $0<\alpha<1$ is done already.

If $\alpha=0$, then $\ell^{1}\left(G, \sigma_{\alpha, C}\right) \cong \ell^{1}(G)$ which is known to be non-Arens regular ( $[5$, Theorem $8.11])$ and so, it is not an operator algebra. Now suppose that $\alpha=1$. For every $m, n \geq 2$, take $a_{m, n} \in F^{m+n} \backslash F^{m+n-1}$ (this is possible because $G$ is infinite). Hence there are $x_{n} \in F^{n}$ and $y_{m} \in F^{m}$ such that

$$
a_{m, n}=x_{n} y_{m} .
$$

Moreover, since $a_{m, n} \in F^{m+n} \backslash F^{m+n-1}$, we have

$$
x_{n} \in F^{n} \backslash F^{n-1} \text { and } y_{m} \in F^{m} \backslash F^{m-1} .
$$

Therefore

Hence

$$
\tau\left(a_{m, n}\right)=m+n, \tau\left(x_{n}\right)=n, \tau\left(y_{m}\right)=m .
$$

$$
\frac{\sigma_{1, C}\left(x_{n} y_{m}\right)}{\sigma_{1, C}\left(x_{n}\right) \sigma_{1, C}\left(y_{m}\right)}=\frac{e^{C \tau\left(x_{n} y_{m}\right)}}{e^{C\left(\tau\left(x_{n}\right)+\tau\left(y_{m}\right)\right)}}=\frac{e^{C m+C n}}{e^{C(n+m)}}=1 \text {. }
$$

Thus

$$
\lim _{n \rightarrow \infty} \lim _{m \rightarrow \infty} \frac{\sigma_{1, C}\left(x_{n} y_{m}\right)}{\sigma_{1, C}\left(x_{n}\right) \sigma_{1, C}\left(y_{m}\right)}=1,
$$

which implies from [5, Theorem 8.11] that $\ell^{1}\left(G, \sigma_{1, C}\right)$ is not Arens regular, and so, it is not an operator algebra.

Remark 3.6. We would like to point out that the upper-bounded estimate obtained in (3.12) goes to $\infty$ as $\alpha$ approaches either 0 or 1 (this happens because $\beta \rightarrow \infty$ ). This coincides with the result obtained in the statement of Theorem 3.5 since as $\alpha \rightarrow 0(\alpha \rightarrow 1$, respectively), the weight $\sigma_{\alpha, C} \rightarrow \sigma_{0, C}=e^{C}\left(\sigma_{\alpha, C} \rightarrow \sigma_{1, C}\right.$, respectively) and we showed there that neither $\ell^{1}\left(G, e^{C}\right)$ nor $\ell^{1}\left(G, \sigma_{1, C}\right)$ is isomorphic to an operator algebra, and so, $\|m\|_{\varepsilon}$ is not bounded.

\section{Remarks on $Q$-Algebras and operator spaCe Versions}

The weighted group algebras in sections 3.1 and 3.2 are injective algebras, but not isomorphic to $Q$-algebras since they are non-commutative in general. However, their algebraic centers are actually isomorphic to $Q$-algebras. Indeed, the injectivity of the tensor product tells us that the algebraic center is also an injective algebra with the smaller norm of the multiplication map. Then, the result in [19] implies that they are isomorphic to $Q$-algebras. Moreover, Theorem 2.6 allows us to determine $(\delta, L)$ for the corresponding multi-variable von Neumann inequality. Thus we have the following. We note that for an algebra $A$, we denote $Z A$ to be its algebraic center.

Corollary 4.1. $Z \ell^{1}\left(G, \omega_{\beta}\right)$ is isomorphic to a $Q$-algebra if one of the following conditions holds:

(i) $\lambda=1$ and $\beta>\frac{d(G)}{2}$;

(ii) $0<\lambda<1$ and $\beta>\frac{d(G)+1}{2}$.

In this case $Z \ell^{1}\left(G, \omega_{\beta}\right)$ satisfy multi-variable $(\delta, L)$-von Neumann inequality with

$$
\delta=e^{-1}\left(1+K_{G} \min \left\{1,2^{\beta-1}\right\}\left[1+\sum_{n=1}^{\infty} \frac{f(n)-\lambda f(n-1)}{(1+n)^{2 \beta}}\right]^{1 / 2}\right)^{-1} \text { and } L=1 .
$$


We have a corresponding result for exponential weights.

Corollary 4.2. Suppose that $0<\alpha<1$ and $C>0$. Then $Z \ell^{1}\left(G, \sigma_{\alpha, C}\right)$ is isomorphic to a $Q$-algebra. In this case, $Z \ell^{1}\left(G, \sigma_{\alpha, C}\right)$ satisfy multi-variable $(\delta, L)$-von Neumann inequality with

$$
\delta=e^{-1}\left(1+K_{G} M 2^{\beta-1}\left[1+\sum_{n=1}^{\infty} \frac{f(n)-\lambda f(n-1)}{(1+n)^{2 \beta}}\right]^{1 / 2}\right)^{-1} \quad \text { and } L=1,
$$

where

$$
\beta=\max \left\{1, \frac{6}{C \alpha(1-\alpha)}, \frac{d+\left(1-\delta_{1}(\lambda)\right)}{2}\right\}
$$

and $M$ is the constant defined in (3.10).

We end this section with a remark on operator space versions. Most of the results in this paper have their operator space versions available following the approach in [8]. For example, the estimates on $\left\|\Omega_{\beta}\right\|_{T^{2}(G)}$ in Theorem 3.1 tells us that $\ell^{1}\left(G, \omega_{\beta}\right)$ with the maximal operator space structure is completely isomorphic to an operator algebra. But in the case of operator spaces we need to show that the algebra multiplication map $m$ extends to a completely bounded maps on the Haagerup tensor product, so that Littlewood multiplier theory has to be developed upto the level of operator spaces as in [8].

\section{EXAMPLES}

5.1. The d-dimensional integers $\mathbb{Z}^{d}$. A usual choice of generating set is

$$
F=\left\{\left(x_{1}, \ldots, x_{d}\right) \mid x_{i} \in\{-1,0,1\}\right\},
$$

It is straightforward to see that

$$
\tau\left(\left(x_{1}, \ldots, x_{d}\right)\right)=\max \left\{\left|x_{1}\right|, \ldots,\left|x_{d}\right|\right\}
$$

and for every $n \in \mathbb{N}$,

$$
F^{n}=\left\{\left(x_{1}, \ldots, x_{d}\right) \mid x_{i} \in\{-n, \ldots, 0, \ldots, n\}\right\} .
$$

Thus we get

$$
\left|F^{n}\right|=(2 n+1)^{d} \quad(n=0,1,2, \ldots)
$$

and the order of growth of $\mathbb{Z}^{d}$ is $d$ with $f(n)=(2 n+1)^{d}$ and $\lambda=1$. It follows from Theorem 3.1 that $\ell^{1}\left(\mathbb{Z}^{d}, \omega_{\beta}\right)$ is isomorphic to an operator algebras if $\beta>\frac{d}{2}$. Moreover, we have

$$
\begin{aligned}
\sum_{n=1}^{\infty} \frac{f(n)-\lambda f(n-1)}{(1+n)^{2 \beta}} & =\sum_{n=1}^{\infty} \frac{(2 n+1)^{d}-(2 n-1)^{d}}{(1+n)^{2 \beta}} \\
& \leq \sum_{n=1}^{\infty} \frac{2 d(2 n+2)^{d-1}}{(1+n)^{2 \beta}}=d 2^{d} \sum_{n=1}^{\infty}(1+n)^{d-1-2 \beta} \\
& \leq d 2^{d} \int_{1}^{\infty} x^{d-1-2 \beta} d x=\frac{d 2^{d}}{2 \beta-d}
\end{aligned}
$$

Since $\mathbb{Z}^{d}$ is an abelian group, Theorem 4.1 tells us that $\ell^{1}\left(\mathbb{Z}^{d}, \omega_{\beta}\right)$ is actually a $Q$-algebra and it satisfies multi-variable von Neumann inequality for $L=1$ and

$$
\delta=e^{-1}\left\{1+K_{G} \min \left\{1,2^{\beta-1}\right\}\left[1+\frac{d 2^{d}}{2 \beta-d}\right]^{1 / 2}\right\}^{-1} .
$$


On the other hand, $\ell^{1}\left(\mathbb{Z}^{d}, \omega_{\beta}\right)$ fails to be an injective algebra if $\beta \leq \frac{d}{2}$ ([8]).

Now let $\sigma_{\alpha, C}$ be the exponential weight on $\mathbb{Z}^{d}$ defined in (2.11). Theorem 4.2 tells us that $\ell^{1}\left(\mathbb{Z}^{d}, \sigma_{\alpha, C}\right)$ is a $Q$-algebra and it satisfies multi-variable von Neumann inequality for $L=1$ and

$$
\delta=e^{-1}\left(1+K_{G} M 2^{\beta-1}\left[1+\frac{d 2^{d}}{2 \beta-d}\right]^{1 / 2}\right)^{-1},
$$

where

$$
\beta=\max \left\{1, \frac{6}{C \alpha(1-\alpha)}, \frac{d}{2}\right\}
$$

and $M$ is the constant defined in (3.10).

A case of particular interest happens when we let

$$
d=1 \quad \text { and } \quad C=\frac{6}{\alpha(1-\alpha)} .
$$

In this case, we can choose $\beta=1$. Also if $K$ is the constant defined in (3.11), then it is easy to see that $0<K<1 / 6$. Hence $M=1$, and so we get

$$
\delta=\frac{1}{e\left(1+\sqrt{3} K_{G}\right)} .
$$

5.2. The 3-dimensional discrete Heisenberg group $\mathbb{H}_{3}(\mathbb{Z})$. We recall that the 3 -dimensional discrete Heisenberg group $\mathbb{H}_{3}(\mathbb{Z})$ is a semidirect product of $\mathbb{Z}^{2}$ with $\mathbb{Z}$ and the product is defined as follows:

$$
\left(a_{1}, b_{1}, c_{1}\right) \cdot\left(a_{2}, b_{2}, c_{2}\right)=\left(a_{1}+a_{2}, b_{1}+b_{2}, a_{1} b_{2}+c_{1}+c_{2}\right) \quad\left(a_{i}, b_{i}, c_{i}\right) \in \mathbb{H}_{3}(\mathbb{Z}) .
$$

If we identify $\mathbb{Z}$ with the subgroup $\{(0,0, c): c \in \mathbb{Z}\}$, then it is easy to see that $\mathbb{H}_{3}(\mathbb{Z}) / \mathbb{Z} \cong \mathbb{Z}^{2}$. Hence $\mathbb{H}_{3}(\mathbb{Z})$ is a 2 -step nilpotent group and by the Bass-Guivarch formula (2.6) we have

$$
d\left(\mathbb{H}_{3}(\mathbb{Z})\right)=4 .
$$

Hence if we let $\omega_{\beta}$ be the polynomial weight on $\mathbb{H}_{3}(\mathbb{Z})$, then, $\ell^{1}\left(\mathbb{H}_{3}(\mathbb{Z}), \omega_{\beta}\right)$ is isomorphic to an operator algebra provided that

$$
\beta>\frac{4+1}{2}=\frac{5}{2}
$$

Moreover, $Z \ell^{1}\left(\mathbb{H}_{3}(\mathbb{Z}), \omega_{\beta}\right)$ satisfies multi-variable von Neumann inequality. On the other hand, the restriction of $\omega_{\beta}$ to $\mathbb{Z}$ will be a weight equivalent to the weight $\omega_{\beta}^{\prime}(c)=(1+|c|)^{\beta}$. Hence $\ell^{1}\left(\mathbb{H}_{3}(\mathbb{Z}), \omega_{\beta}\right)$ has a closed subalgebra which is isomorphic to $\ell^{1}\left(\mathbb{Z}, \omega_{\beta}^{\prime}\right)$. Thus it follows from the result of Varopoulos [19] that $\ell^{1}\left(\mathbb{H}_{3}(\mathbb{Z}), \omega_{\beta}\right)$ fails to be an injective algebra if $\beta \leq 1 / 2$.

5.3. The free group with two generators $\mathbb{F}_{2}$. In this subsection we will show that $\ell^{1}\left(\mathbb{F}_{2}, \omega_{\beta}\right)$ is not an injective algebra for any $\beta>0$. Since $\mathbb{F}_{2}$ is one of the typical examples of exponentially growing groups, this gives evidence to suggest that the condition of polynomial growth on the group is necessary for a weighted group to be realizable as an operator algebras.

Recall also the Rudin-Shapiro polynomials defined in the following recursive way ([2, Chapter 4]).

$$
P_{0}(z):=1, \quad Q_{0}(z):=1
$$

and for $k \geq 0$

$$
P_{k+1}(z):=P_{k}(z)+z^{2^{k}} Q_{k}(z), \quad Q_{k+1}(z):=Q_{k}(z)-z^{2^{k}} P_{k}(z)
$$


By an induction on $k$, it is straightforward to check that the coefficients of $P_{k}$ are $\pm 1, \operatorname{deg} P_{k}=$ $\operatorname{deg} Q=2^{k}-1$ and

$$
\left|P_{k}(z)\right|^{2}+\left|Q_{k}(z)\right|^{2}=2^{k+1} \quad(z \in \mathbb{T}) .
$$

Hence

$$
\left\|P_{k}\right\|_{L^{\infty}(\mathbb{T})} \leq \sqrt{2^{k+1}}
$$

Using the following contraction (actually it is a metric surjection due to Nehari's theorem, see [14, Section 6] for example)

$$
Q: L^{\infty}(\mathbb{T}) \rightarrow B\left(\ell^{2}\right), \quad f \mapsto(\widehat{f}(-(i+j)))_{i, j \in \mathbb{Z}}
$$

we get a sequence of Hankelian matrices

$$
A_{2^{k}}=Q\left(\bar{P}_{k}\right), k \geq 0,
$$

where $A_{2^{k}}$ is a $2^{k} \times 2^{k}$ matrix with entries \pm 1 satisfying

$$
\left\|A_{2^{k}}\right\|_{\text {op }} \leq \sqrt{2^{k+1}}
$$

where $\|\cdot\|_{\text {op }}$ means the operator norm.

Theorem 5.1. $\ell^{1}\left(\mathbb{F}_{2}, \omega_{\beta}\right)$ is not an injective algebra for any $\beta>0$.

Proof. Let $g_{1}$ and $g_{2}$ be two generators of $\mathbb{F}_{2}$, and let $d$ be an even positive integer with $d>2 \beta$. Consider the following subsets of $\mathbb{F}_{2}$.

$$
I_{n}^{d}=\left\{g_{1}^{x_{1}} g_{2}^{x_{2}} g_{1}^{x_{3}} \cdots g_{2}^{x_{d}}: 1 \leq x_{i} \leq n \text { for } i=1, \ldots, d\right\},
$$

Now we recall the function $\Omega_{\beta}$ defined by

$$
\Omega_{\beta}\left(g, g^{\prime}\right)=\frac{\omega_{\beta}\left(g g^{\prime}\right)}{\omega_{\beta}(g) \omega_{\beta}\left(g^{\prime}\right)}, \quad\left(g, g^{\prime} \in \mathbb{F}\right) .
$$

Let $\Omega_{\beta}^{n}=\Omega_{\beta} 1_{I_{n}^{d} \times I_{n}^{d}}$. When $g, g^{\prime} \in I_{n}^{d}$ are given by $g=g_{1}^{x_{1}} g_{2}^{x_{2}} g_{1}^{x_{3}} \cdots g_{2}^{x_{d}}$ and $g^{\prime}=g_{1}^{y_{1}} g_{2}^{y_{2}} g_{1}^{y_{3}} \cdots g_{2}^{y_{d}}$ for $x_{i}, y_{j} \geq 1$, then we have

$$
\Omega_{\beta}^{n}\left(g, g^{\prime}\right)=\left(\frac{1+x_{1}+\cdots+x_{n}+y_{1}+\cdots+y_{n}}{\left(1+x_{1}+\cdots+x_{n}\right)\left(1+y_{1}+\cdots+y_{n}\right)}\right)^{\beta} .
$$

By a similar estimate as in [8, Theorem 6.1$]$ we get

$$
\left\|\Omega_{\beta}^{n}\right\|_{\mathrm{op}} \geq 2^{-\beta} n^{\frac{d}{2}}\left(\sum_{1 \leq x_{1}, \cdots, x_{d} \leq n} \frac{1}{\left(1+x_{1}+\cdots+x_{d}\right)^{2 \beta}}\right)^{\frac{1}{2}}
$$

Now using the Rudin-Shapiro polynomial, we have a sequence of matrices $A_{n} \in M_{n}, n=2^{k}$ $(k=1,2, \ldots)$ satisfying the following conditions:

(1) $A_{n}=\left(a_{i+j}^{n}\right)_{i, j=1}^{n}$ with $a_{i}^{n} \in\{ \pm 1\}$.

(2) $\left\|A_{n}\right\|_{\text {op }} \leq \sqrt{2 n}$.

We consider $b=\left(b_{h}\right)_{h \in \mathbb{F}_{2}}$ given by

$$
\begin{cases}b_{g g^{\prime}}=a_{x_{1}+y_{1}}^{n} \cdots a_{x_{d}+y_{d}}^{n} & \text { for } g=g_{1}^{x_{1}} g_{2}^{x_{2}} g_{1}^{x_{3}} \cdots g_{2}^{x_{d}}, g^{\prime}=g_{1}^{y_{1}} g_{2}^{y_{2}} g_{1}^{y_{3}} \cdots g_{2}^{y_{d}}, x_{i}, y_{j} \geq 1 \\ b_{h}=0 & \text { elsewhere. }\end{cases}
$$


In other words, the matrix $\left[b_{g g^{\prime}}\right]_{g, g^{\prime} \in I_{n}^{d}}$ is nothing but the $d$-tensor power of the matrix $\left[a_{x+y}^{n}\right]_{1 \leq x, y \leq n}$. Thus it follows from [18, Theorem 3.1 and Corollary 3.2], (3.1) and $\|b\|_{\text {op }} \leq(2 n)^{\frac{d}{2}}$ that

$$
\begin{aligned}
\|\widetilde{\Gamma}\| & \geq\|\widetilde{\Gamma}(b)\|_{\left(\ell^{1}(G) \otimes_{\varepsilon} \ell^{1}(G)\right)^{*}}=\|\Gamma(b) \Omega\|_{\left(\ell^{1}(G) \otimes_{\varepsilon} \ell^{1}(G)\right)^{*}} \\
& \geq K_{G}^{-1}\|\Gamma(b) \Omega\|_{\left(\ell^{1}(G) \otimes_{h} \ell^{1}(G)\right)^{*}}=K_{G}^{-1}\left\|\left[b_{g g^{\prime}} \Omega_{\beta}^{n}\left(g, g^{\prime}\right)\right]_{g, g^{\prime} \in I_{n}^{d}}\right\|_{\text {Schur }} \\
& \left.\geq K_{G}^{-1}\left\|\left[b_{g g^{\prime}}\right]_{g, g^{\prime} \in I_{n}^{d}}\right\|_{\text {op }}^{-1}\left\|\Omega_{\beta}^{n}\right\|_{\mathrm{op}}\right)^{\frac{1}{2}} \\
& \geq K_{G}^{-1}(2 n)^{-\frac{d}{2}} 2^{-\beta} n^{\frac{d}{2}}\left(\sum_{1 \leq x_{1}, \cdots, x_{d} \leq n} \frac{1}{\left(1+x_{1}+\cdots+x_{d}\right)^{2 \beta}}\right)^{\frac{1}{2}} \\
& =K_{G}^{-1} 2^{-\frac{d}{2}} 2^{-\beta}\left(\sum_{1 \leq x_{1}, \cdots, x_{d} \leq n} \frac{1}{\left(1+x_{1}+\cdots+x_{d}\right)^{2 \beta}}\right)^{-\cdots} \\
& \longrightarrow \infty \text { as } n=2^{k} \rightarrow \infty \text { since } 2 \beta<d .
\end{aligned}
$$

Hence $\ell^{1}\left(\mathbb{F}_{2}, \omega_{\beta}\right)$ is not an injective algebra for any $\beta>0$. Note that in $(*)$, we are using the fact that the Schur product of $\left[b_{g g^{\prime}}\right]$ with itself is the matrix with all entries 1 which is the identity in the Schur product.

\section{REFERENCES}

[1] H. Bass, The degree of polynomial growth of finitely generated nilpotent groups, Proc. London Math. Soc. (3) 25 (1972), 603-614.

[2] P. Borwein, Computational excursions in analysis and number theory. (English summary) CMS Books in Mathematics/Ouvrages de Mathmatiques de la SMC, 10. Springer-Verlag, New York, 2002.

[3] J. Brita, H. Buhrmana, T. Leeb, T. Vidickc, All Schatten spaces endowed with the Schur product are Qalgebras, J. Funct. Anal. 262, Issue 1, 2012, 1-9.

[4] D. Blecher and C. Le Merdy, Operator algebras and their modules-an operator space approach, London Mathematical Society Monographs. New Series, 30. Oxford Science Publications. The Clarendon Press, Oxford University Press, Oxford, 2004.

[5] H. G. Dales and A. T.-M. Lau, The Second Duals of Beurling Algebras, Mem. Amer. Math. Soc, 177 n. 836 (2005).

[6] A. M. Davie, Quotient algebras of uniform algebras, J. London Math. Soc. (2) 7 (1973), 31-40.

[7] J. Diestel, H. Jarchow, A. Tonge, Absolutely summing operators. Cambridge Studies in Advanced Mathematics, 43. Cambridge University Press, Cambridge, 1995. xvi+474 pp.

[8] M. Ghandehari, H.H. Lee, E. Samei and N. Spronk, Some Beurling-Fourier algebras are operator algebras. preprint.

[9] M. Gromov, Groups of Polynomial growth and Expanding Maps, Inst. Hautes Études Sci. Publ. Math. No. 53, 1981, 53-73.

[10] Y. Guivarch, Groupes de Lie à croissance polynomiale, C. R. Acad. Sci. Paris Sér. A-B, 272 (1971), A1695A1696.

[11] C. Le Merdy, The Schatten space $S_{4}$ is a Q-algebra, Proc. Amer. Math. Soc. 126 (1998), no. 3, 715-719.

[12] T. W. Palmer, Banach algebras and general theorem of *algebras, II. Cambrige University Press, 2001.

[13] D. Pérez-García, The trace class is a Q-algebra. Ann. Acad. Sci. Fenn. Math. 31 (2006), no. 2, 287295.

[14] G. Pisier, Similarity problems and completely bounded maps. Second, expanded edition. Includes the solution to "The Halmos problem". Lecture Notes in Mathematics, 1618. Springer-Verlag, Berlin, 2001.

[15] G. Pisier, Introduction to Operator Space Theorey, Cambridge University Press, 2003.

[16] G. Pisier, Grothendieck's theorem, past and present., Bull. Amer. Math. Soc. (N.S.) 49 (2012), no. 2, $237-323$.

[17] R.A. Ryan, Introduction to tensor products of Banach spaces. Springer Monographs in Mathematics. SpringerVerlag London, Ltd., London, 2002. 
[18] N. Spronk, Measurable Schur multipliers and completely bounded multipliers of the Fourier algebras, Proc. London Math. Soc. (3) 89 (2004), no. 1, 161-192.

[19] N.T. Varopoulos, Some remarks on Q-algebras, Ann. Inst. Fourier (Grenoble) 22 (1972), no. 4, 1-11.

[20] N.T. Varopoulos, Sur les quotients des algèbres uniformes. (French) C. R. Acad. Sci. Paris Sr. A-B 274 (1972), A1344-A1346.

Department of Mathematical Sciences, Seoul National University, San56-1 ShinRim-dong KwanakGu SEOul 151-747, Republic of Korea

E-mail address: hhlee@chungbuk.ac.kr

Department of Mathematics and Statistics, University of Saskatchewan, Saskatoon, Saskatchewan, S7N 5E6, CANADA

E-mail address: samei@math.usask.ca

Department of Pure Mathematics, University of Waterloo, Waterloo, Ontario, N2L 3G1, Canada

E-mail address: nspronk@math.uwaterloo.ca 